\title{
Precise model of Hawking radiation from the tunnelling mechanism
}

\author{
Christian Corda \\ July 19, 2018 \\ Dipartimento di Fisica, Scuola Superiore di Studi Universitari e Ricerca \\ "Santa Rita", via Trasaghis 18/E, 00188 Roma, Italy
}

Austro-Ukrainian Institute for Science and Technology, Institut für

Theoretishe Physik, Technische Universität, Wiedner Hauptstrasse 8-10/136, A-1040, Wien, Austria

International Institute for Applicable Mathematics \& Information Sciences (IIAMIS), B.M. Birla Science Centre, Adarsh Nagar, Hyderabad - 500 463, India

E-mail address: cordac.galilei@gmail.com

\begin{abstract}
We recently improved the famous result of Parikh and Wilczek, who found a probability of emission of Hawking radiation which is compatible with a non-strictly thermal spectrum, showing that such a probability of emission is really associated to two non-strictly thermal distributions for boson and fermions. Here we finalize the model by finding the correct value of the pre-factor of the Parikh and Wilczek probability of emission. In fact, that expression has the $\sim$ sign instead of the equality. In general, in this kind of leading order tunnelling calculations, the exponent arises indeed from the classical action and the pre-factor is an order Planck constant correction. But in the case of emissions of Hawking quanta, the variation of the Bekenstein-Hawking entropy is order 1 for an emitted particle having energy of order the Hawking temperature. As a consequence, the exponent in the Parikh and Wilczek probability of emission is order unity and one asks what is the real significance of that scaling if the pre-factor is unknown. Here we solve the problem assuming the unitarity of the black
\end{abstract}


hole $(\mathrm{BH})$ quantum evaporation and considering the natural correspondence between Hawking radiation and quasi-normal modes (QNMs) of excited BHs, in a "Bohr-like model" that we recently discussed in a series of papers. In that papers, QNMs are interpreted as natural $\mathrm{BH}$ quantum levels (the "electron states" in the "Bohr-like model"). Here we find the intriguing result that, although in general it is well approximated by 1 , the pre-factor of the Parikh and Wilczek probability of emission depends on the $\mathrm{BH}$ quantum level $n$. We also write down an elegant expression of the probability of emission in terms of the BH quantum levels.

Hawking radiation [1] is today studied in an elegant and largely used way through the tunnelling mechanism, see [2, 3, 4, 5, 6, 7, 8, 41] and refs. within. Let us see how that mechanism works. One considers an object which is classically stable. If it becomes unstable from a quantum-mechanically point of view, one naturally suspects tunnelling. Hawking's famous mechanism of particles creation by $\mathrm{BH}$ [1] is, in turn, described in terms of tunnelling arising from vacuum fluctuations near the $\mathrm{BH}$ horizon $[2,3,4,5,6,6,7,8,41$. When a virtual particle pair is created just inside the $\mathrm{BH}$ horizon, the virtual particle having positive energy can tunnel out. Thus, it materializes outside the $\mathrm{BH}$ as a real particle. In analogous way, when a virtual particle pair is created just outside the horizon, the particle having negative energy can tunnel inwards. In both cases, the particle having negative energy is absorbed by the $\mathrm{BH}$. The result is that the $\mathrm{BH}$ mass decreases and the particle having positive energy propagates towards infinity. Thus, subsequent emissions of Hawking quanta appear.

Working with $G=c=k_{B}=\hbar=\frac{1}{4 \pi \epsilon_{0}}=1$ (Planck units), in strictly thermal approximation the probability of emission of Hawking quanta is [1, 2, 3, 9]

$$
\Gamma \sim \exp \left(-\frac{\omega}{T_{H}}\right),
$$

where $\omega$ is the energy-frequency of the emitted particle and $T_{H} \equiv \frac{1}{8 \pi M}$ is the Hawking temperature. Taking into account the energy conservation, i.e. the $\mathrm{BH}$ contraction enabling a varying $\mathrm{BH}$ geometry, one gets the remarkable correction of Parikh and Wilczek [2, 3]

$$
\Gamma \sim \exp \left[-\frac{\omega}{T_{H}}\left(1-\frac{\omega}{2 M}\right)\right] \quad \Longrightarrow \quad \Gamma=\alpha \exp \left[-\frac{\omega}{T_{H}}\left(1-\frac{\omega}{2 M}\right)\right],
$$

where $\alpha \sim 1$ and the additional term $\frac{\omega}{2 M}$ is present. We have recently improved the Parikh and Wilczek tunnelling picture showing that the probability of emission (2) is, indeed, associated to the two distributions [8]

$$
\begin{aligned}
<N>_{\text {boson }}=\frac{1}{\exp [4 \pi(2 M-\omega) \omega]-1} \\
<N>_{\text {fermion }}=\frac{1}{\exp [4 \pi(2 M-\omega) \omega]+1},
\end{aligned}
$$

for bosons and fermions respectively, which are non strictly thermal.

We note that eq. (2) has the $\sim$ sign instead of the equality. In fact, in this kind of leading order tunnelling calculations the exponent arises from the 
classical action and the pre-factor is an order Planck constant correction. But in the case of emissions of Hawking quanta the variation of the Bekenstein-Hawking entropy [2, 3]

$$
\Gamma=\alpha \exp \Delta S_{B H}=\alpha \exp \left[-\frac{\omega}{T_{H}}\left(1-\frac{\omega}{2 M}\right)\right],
$$

is order 1 for an emitted particle having energy of order the Hawking temperature. As a consequence, the exponent in the right hand side of eqs. (2) and (4) is order unity and we ask what is the real significance of that scaling if pre-factor is unknown. Here we solve the problem considering the natural correspondence between Hawking radiation and BH QNMs, in a "Bohr-like model" that we recently discussed in a series of papers [9, 10, 11, 12, also together with collaborators [13, 14].

We consider Dirac delta perturbations [9, 10, 11, 12, which represent subsequent absorptions of particles having negative energies. Such perturbations are associated to emissions of Hawking quanta in the above discussed mechanism of particle pair creation. BH response to perturbations are QNMs [9, 10, 11, 12, 13, [14, 15, 16, which are frequencies of radial spin- $j$ perturbations obeying a time independent Schröedinger-like equation [9, 10, 11, 12, 16]. They are the BH modes of energy dissipation which frequency is allowed to be complex [9, 10, 11, 12, 16]. The intriguing idea to model the quantum BH in terms of BH QNMs arises from a remarkable paper by York [17. For large values of the quantum "overtone" number $n$, where $n=1,2, \ldots$, QNMs become independent of both the spin and the angular momentum quantum numbers $99,10,11,12,15,16$, in perfect agreement with Bohr's Correspondence Principle [18, which states that "transition frequencies at large quantum numbers should equal classical oscillation frequencies". Thus, Bohr's Correspondence Principle enables an accurate semi-classical analysis for large values of the principal quantum number $n$, i.e, for excited BHs. By using that principle, Hod has shown that QNMs release information about the area quantization as QNMs are associated to absorption of particles [19, 20]. Hod's work was refined by Maggiore [15] who solved some important problems. On the other hand, as QNMs are countable frequencies, ideas on the continuous character of Hawking radiation did not agree with attempts to interpret QNMs in terms of emitted quanta, preventing to associate QNMs to Hawking radiation [16]. Recently, the authors of [21, 22, 23, 24] and ourselves and collaborators [10, 11, 12, 13, 14, observed that the non-thermal spectrum of Parikh and Wilczek [2, 3] also implies the countable character of subsequent emissions of Hawking quanta. This issue enables a natural correspondence between QNMs and Hawking radiation, permitting to interpret QNMs also in terms of emitted energies [10, 11, 12, 13, 14. Dirac delta perturbations due to discrete subsequent absorptions of particles having negative energies, which are associated to emissions of Hawking quanta in the mechanism of particle pair creation by quantum fluctuations, generates indeed BH QNMs [10, 11, 12, 13, 14. In other words, the $\mathrm{BH}$ contraction due to the energy conservation is not a "one shot process". It generates oscillations of the horizon instead, which are the QNMs. We also stress that the correspondence between emitted radiation and proper oscillation of the emitting body is a fundamental behavior of every radiation 
process in science. Based on such a natural correspondence between Hawking radiation and $\mathrm{BH}$ QNMs, one can consider QNMs in terms of quantum levels also for emitted energies [10, 11, 12, 13, 14].

Let us see how the model works. By introducing the effective temperature [8, 10, 11, 12

$$
T_{E}(\omega) \equiv \frac{2 M}{2 M-\omega} T_{H}=\frac{1}{4 \pi(2 M-\omega)},
$$

one rewrites eq. (5) in a Boltzmann-like form similar to eq. (1)

$$
\Gamma=\alpha \exp \left[-\beta_{E}(\omega) \omega\right]=\alpha \exp \left(-\frac{\omega}{T_{E}(\omega)}\right),
$$

where $\exp \left[-\beta_{E}(\omega) \omega\right]$ is the effective Boltzmann factor, with $\beta_{E}(\omega) \equiv \frac{1}{T_{E}(\omega)}$. Thus, the effective temperature replaces the Hawking temperature in the equation of the probability of emission [8, 10, 11, 12. We emphasize that there are various fields of science where one takes into account the deviation from the thermal spectrum of an emitting body by introducing an effective temperature which represents the temperature of a black body that would emit the same total amount of radiation. We introduced the concept of effective temperature in $\mathrm{BH}$ physics in [10, 11] and used it in [8, 10, 11, 12] and, together with collaborators, in 13, 14. The effective temperature depends on the energy-frequency of the emitted radiation and the ratio $\frac{T_{E}(\omega)}{T_{H}}=\frac{2 M}{2 M-\omega}$ represents the deviation of the $\mathrm{BH}$ radiation spectrum from the strictly thermal feature [8, 10, 11, 12]. The introduction of the effective temperature permits the introduction of other effective quantities. Considering the initial $\mathrm{BH}$ mass before the emission, $M$, and the final $\mathrm{BH}$ mass after the emission, $M-\omega$, one introduces the $B H$ effective mass and the BH effective horizon [8, 10, 11, 12] as

$$
M_{E} \equiv M-\frac{\omega}{2}, r_{E} \equiv 2 M_{E},
$$

during the $\mathrm{BH}$ contraction, i.e. during the emission of the particle [10-12]. Such effective quantities are average quantities [8, 10, 11, 12]. In fact, $r_{E}$ is the average of the initial and final horizons while $M_{E}$ is the average of the initial and final masses [8, 10, 11, 12]. The effective temperature $T_{E}$ is the inverse of the average value of the inverses of the initial and final Hawking temperatures (before the emission $T_{H}$ initial $=\frac{1}{8 \pi M}$, after the emission $T_{H}$ final $=\frac{1}{8 \pi(M-\omega)}$ ) [8, 10, 11, 12.

For large values of the principal quantum number $n$, i.e, for excited BHs, and independently of the angular momentum quantum number, the QNMs expression of the Schwarzschild BH which takes into account the non-strictly thermal behavior of the radiation spectrum is obtained replacing the Hawking temperature with the effective temperature in the standard, strictly thermal, equation for the quasi-normal frequencies as $8,10,11,12$,

$$
\begin{aligned}
& \omega_{n}=a+i b+2 \pi i n \times T_{E}\left(\left|\omega_{n}\right|\right) \\
& \simeq 2 \pi i n \times T_{E}\left(\left|\omega_{n}\right|\right)=\frac{i n}{4 M-2\left|\omega_{n}\right|},
\end{aligned}
$$


where $a$ and $b$ are real numbers with $a=(\ln 3) \times T_{E}\left(\left|\omega_{n}\right|\right), b=\pi \times T_{E}\left(\left|\omega_{n}\right|\right)$ for $j=0,2$ (scalar and gravitational perturbations), $a=0, b=0$ for $j=1$ (vector perturbations) and $a=0, b=\pi \times T_{E}\left(\left|\omega_{n}\right|\right)$ for half-integer values of $j$. On the other hand, as $a, b \ll\left|2 \pi i n T_{E}\left(\left|\omega_{n}\right|\right)\right|$, a fundamental consequence is that the quantum of area obtained from the asymptotics of $\left|\omega_{n}\right|$ is an intrinsic property of Schwarzschild BHs because for large $n$ the leading asymptotic behavior of $\left|\omega_{n}\right|$ is given by the leading term in the imaginary part of the complex frequencies, and it does not depend on the spin content of the perturbation [10, 11, 12, 15]. An intuitive derivation of eq. (8) can be found in [10, 11]. We rigorously derived such an equation in the Appendix of [12. Further important clarifications on the derivation of eq. (8) have been highlighted in the recent review paper 37 through Hawking's periodicity arguments 38 .

Eq. (8) has the following elegant interpretation [10, 11. The quasi-normal frequencies determine the position of poles of a Green's function on the given background, and the Euclidean $\mathrm{BH}$ solution converges to a non-strictly thermal circle at infinity with the inverse temperature $\beta_{E}\left(\omega_{n}\right)=\frac{1}{T_{E}\left(\left|\omega_{n}\right|\right)}$ 10, 11. Thus, the spacing of the poles in eq. (8) coincides with the spacing $2 \pi i T_{E}\left(\left|\omega_{n}\right|\right)=$ $2 \pi i T_{H}\left(\frac{2 M}{2 M-\left|\omega_{n}\right|}\right)$, expected for a non-strictly thermal Green's function [10, 11]. We found the physical solution for the absolute values of the frequencies (8) in [10, 11, 12. Considering the leading asymptotic behavior one gets 10, 11, 12,

$$
E_{n} \equiv\left|\omega_{n}\right|=M-\sqrt{M^{2}-\frac{n}{2}} .
$$

$E_{n}$ is interpreted like the total energy emitted by the $\mathrm{BH}$ at that time, i.e. when the $\mathrm{BH}$ is excited at a level $n$ [10, 11, 12. Considering an emission from the ground state (i.e. a $\mathrm{BH}$ which is not excited) to a state with large $n=n_{1}$ and using eq. (9), the $\mathrm{BH}$ mass changes from $M$ to [10, 11, 12]

$$
M_{n_{1}} \equiv M-E_{n_{1}}=\sqrt{M^{2}-\frac{n_{1}}{2}} .
$$

In the transition from the state with $n=n_{1}$ to a state with $n=n_{2}$ where $n_{2}>n_{1}$ the $\mathrm{BH}$ mass changes again from $M_{n_{1}}$ to

$$
\begin{gathered}
M_{n_{2}} \equiv M_{n_{1}}-\Delta E_{n_{1} \rightarrow n_{2}}=M-E_{n_{2}} \\
=\sqrt{M^{2}-\frac{n_{2}}{2}}
\end{gathered}
$$

where

$$
\Delta E_{n_{1} \rightarrow n_{2}} \equiv E_{n_{2}}-E_{n_{1}}=M_{n_{1}}-M_{n_{2}}=\sqrt{M^{2}-\frac{n_{1}}{2}}-\sqrt{M^{2}-\frac{n_{2}}{2}},
$$

is the jump between the two levels due to the emission of a particle having frequency $\Delta E_{n_{1} \rightarrow n_{2}}$. Thus, in our $\mathrm{BH}$ model [12], during a quantum jump a discrete amount of energy is radiated and, for large values of the principal quantum number $n$, the analysis becomes independent of the other quantum numbers. In a certain sense, QNMs represent the "electron" which jumps from a level to another one and the absolute values of the QNMs frequencies represent 
the energy "shells" [2]. In Bohr model of the hydrogen atom [25, 26] electrons can only gain and lose energy by jumping from one allowed energy shell to another, absorbing or emitting radiation with an energy difference of the levels according to the Planck relation (in standard units) $E=h f$, where $h$ is the Planck constant and $f$ the transition frequency. In our Bohr-like $\mathrm{BH}$ model 10, 11, 12, QNMs can only gain and lose energy by jumping from one allowed energy shell to another, absorbing or emitting radiation (emitted radiation is given by Hawking quanta) with an energy difference of the levels according to eq. (12). The similarity is completed if one notes that the interpretation of eq. (9) is of a particle, the "electron", quantized on a circle of length [10, 11]

$$
L=\frac{1}{T_{E}\left(E_{n}\right)}=4 \pi\left(M+\sqrt{M^{2}-\frac{n}{2}}\right)
$$

which is the analogous of the electron travelling in circular orbits around the hydrogen nucleus, similar in structure to the solar system, of Bohr model [25, 26]. On the other hand, Bohr model is an approximated model of the hydrogen atom with respect to the valence shell atom model of full quantum mechanics. In the same way, our Bohr-like BH model [12] should be an approximated model with respect to the definitive, but at the present time unknown, $\mathrm{BH}$ model arising from a full quantum gravity theory.

As $E_{n}$ is interpreted like the total energy emitted at level $n$ [12], considering the expressions (10) and (11) for the residual BH mass one needs also [12]

$$
M^{2}-\frac{n}{2} \geq 0
$$

In fact, $\mathrm{BHs}$ cannot emit more energy than their total mass and the total energy emitted by the $\mathrm{BH}$ cannot be imaginary. The expression (14) gives a maximum value for the overtone number $n$

$$
n \leq n_{\max }=2 M^{2},
$$

which corresponds to $E_{n_{\max }}=M$. On the other hand, we recall that, by using the Generalized Uncertainty Principle, Adler, Chen and Santiago [27] have shown that the total $\mathrm{BH}$ evaporation is prevented in exactly the same way that the Uncertainty Principle prevents the hydrogen atom from total collapse. In fact, the collapse is prevented, not by symmetry, but by dynamics, as the Planck distance and the Planck mass are approached [27]. That important result implies that eq. (14) has to be slightly modified, becoming (the Planck mass is equal to 1 in Planck units) [12]

$$
M^{2}-\frac{n}{2} \geq 1
$$

Thus, one gets a slightly different value of the maximum value of the overtone number $n$

$$
n \leq n_{\max }=2\left(M^{2}-1\right) .
$$


Then, the countable sequence of QNMs for emitted energies cannot be infinity although $n$ can be extremely large [12]. In fact, restoring ordinary units and considering a $\mathrm{BH}$ mass of the order of 10 solar masses, one easily gets $n_{\max } \sim 10^{76}$. On the other hand, we expect further corrections to our semi-classical analysis when the Planck scale is approached, as we need a full theory of quantum gravity to obtain a correct description of the Planck scale's physics.

Our Bohr-like BH model in [10, 11, 12] is in full agreement with previous literature of $\mathrm{BH}$ thermodynamics, like references [15, 28, 29]. More, it is also in full agreement with the famous result of Bekenstein on the area quantization [30]. In fact, we found an area quantum arising from a jump among two neighbouring quantum levels $n-1$ and $n$ having a value $\left|\triangle A_{n}\right|=\left|\triangle A_{n-1}\right| \simeq 8 \pi$, see eq. (37) in [12, which is totally consistent with Bekenstein's result 30. Clearly, all these similarities with the Bohr semi-classical model of the hydrogen atom and all these consistences with well known results in the literature of BHs, starting by the universal Bekenstein's result, cannot be coincidences, but are confirmations of the correctness of the analysis in [10, 11, 12] instead.

Concerning the important issue of the $\mathrm{BH}$ entropy, we recall the recent interesting result in which the entropy is connected with the tunnelling mechanism [40, 42]. Jiang and Han [40] have indeed quantized the BH entropy by combining the proposal about the $\mathrm{BH}$ adiabatic invariance and the proposal about the oscillating velocity of the $\mathrm{BH}$ horizon, where the velocity is obtained in the tunneling framework.

Now, let us proceed in calculating the correct value of the pre-factor of eqs. (2) and (44). We recall that, today, the majority of researchers thinks that $\mathrm{BH}$ quantum evaporation is an unitary process and that Hawking's original claim on the information loss in BH evaporation [9] was wrong. Various approaches are indeed proposed by various researchers in order to solve the $\mathrm{BH}$ information paradox and to recover unitarity in $\mathrm{BH}$ evaporation. Here we recall: i) the approach of 21, 22, 23, 24] where the authors found the existence of correlations among Hawking radiation which are elegantly described as hidden messengers in $\mathrm{BH}$ evaporation permitting to restore unitarity in gravitational collapse; ii) the famous ADS/CFT correspondence [31, which was endorsed by both Susskind 32] and Hawking [33], who reversed his opinion in 2004 and recently claimed that BH evaporation is unitary [34; iii) the approach of the so called "fuzzballs" 35]; iv) our recent approach based on the time evolution of our Bohr-like BH model [39]. We have indeed shown in [39] that the time evolution of our Bohrlike BH model is governed by a time dependent Schrödinger equation for the system composed by Hawking radiation and BH QNMs. The physical state and the correspondent wave function are written in terms of an unitary evolution matrix instead of a density matrix [39]. In that way, the final state results to be a pure quantum state instead of a mixed one [39. The approach in [39] permits also to solve the entanglement problem connected with the information paradox because emitted Hawking quanta results to be entangled with BH QNMs. Thus, hereafter we will assume the unitarity of $\mathrm{BH}$ quantum evaporation. Following 39, now we show that, fixed two quantum levels $m$ and $n$, the energy emitted in an arbitrary transition $m \rightarrow n$, with $n>m$, is proportional to the effective 
temperature associated to the transition and that the constant of proportionality depends only on the difference $m-n$. Setting [39]

$$
\Delta E_{m \rightarrow n} \equiv E_{n}-E_{m}=M_{m}-M_{n}=K\left[T_{E}\right]_{m \rightarrow n},
$$

where $M_{m}$ and $M_{n}$ are given by eqs. (10) and (11), let us see if there are values of the constant $K$ for which eq. (18) is satisfied. We recall that [39]

$$
\left[T_{E}\right]_{m \rightarrow n}=\frac{1}{4 \pi\left(M_{m}+M_{n}\right)},
$$

because the effective temperature is the inverse of the average value of the inverses of the initial and final Hawking temperatures, see the above discussion . Thus, eq. (18) can be rewritten as [39]

$$
M_{m}^{2}-M_{n}^{2}=\frac{K}{4 \pi} .
$$

By using eqs. (10) and (11), eq. (20) becomes [39

$$
\frac{1}{2}(n-m)=\frac{K}{4 \pi},
$$

which implies that eq. (18) is satisfied for $K=2 \pi(n-m)$. Hence, one finds 39

$$
\Delta E_{m \rightarrow n}=E_{n}-E_{m}=2 \pi(n-m)\left[T_{E}(\omega)\right]_{m \rightarrow n} .
$$

Using eq. (6), the probability of emission between the two levels $n$ and $m$ can be written in the intriguing form [39]

$$
\Gamma_{m \rightarrow n}=\alpha \exp -\left\{\frac{\Delta E_{m \rightarrow n}}{\left[T_{E}(\omega)\right]_{m \rightarrow n}}\right\}=\alpha \exp [-2 \pi(n-m)] .
$$

Thus, the probability of emission between two arbitrary levels characterized by the two "overtone" quantum numbers $m$ and $n$ scales like $\exp [-2 \pi(n-m)]$. In particular, for $n=m+1$ the probability of emission has its maximum value $\sim \exp (-2 \pi)$, i.e. the probability is maximum for two adjacent levels, as one can intuitively expect [39. If one fixes $m$, the assumption of unitarity in $\mathrm{BH}$ evaporation permits the probabilities (23) to be normalized to the unity as

$$
\sum_{n=m}^{n_{\max }} \Gamma_{m \rightarrow n}=\sum_{n=m}^{n_{\max }} \alpha \exp [-2 \pi(n-m)]=1,
$$

where $n_{\max }$ is the maximum value for the "overtone" number $n$ given by eq. (17) and $n=m$ corresponds to the probability that the $\mathrm{BH}$ does not emit. Putting $k=n-m$ and $\exp [-2 \pi]=X$ eq. (24) becomes

$$
\sum_{k=0}^{k_{\max }} \Gamma_{0 \rightarrow k}=\alpha \sum_{k=0}^{k_{\max }} X^{k}=1
$$


The sum in eq. (25) is the $k t h$ partial sum of the geometric series and can be solved as 36 ]

$$
\sum_{k=0}^{k_{\max }} X^{k}=\frac{1-X^{\left(k_{\max }+1\right)}}{1-X} .
$$

Thus, one gets

$$
\alpha \frac{1-X^{\left(k_{\max }+1\right)}}{1-X}=1,
$$

which permits to solve for $\alpha$

$$
\alpha \equiv \alpha_{m}=\frac{1-X}{1-X^{\left(k_{\max }+1\right)}}=\frac{1-\exp [-2 \pi]}{1-\exp \left[-2 \pi\left(n_{\max }-m+1\right)\right]} .
$$

Hence, we find that the pre-factor $\alpha$ depends on the $\mathrm{BH}$ quantum level $m$. Inserting the result (28) in eq. (23) we fix the probability of emission between the two levels $m$ and $n$ as

$$
\begin{aligned}
\Gamma_{m \rightarrow n} & =\alpha_{m} \exp -\left\{\frac{\Delta E_{m \rightarrow n}}{\left[T_{E}(\omega)\right]_{m \rightarrow n}}\right\}=\alpha_{m} \exp [-2 \pi(n-m)]= \\
& =\left\{\frac{1-\exp [-2 \pi]}{1-\exp \left[-2 \pi\left(n_{\max }-m+1\right)\right]}\right\} \exp [-2 \pi(n-m)] .
\end{aligned}
$$

From the quantum mechanical point of view, one can physically interpret Hawking radiation like energies of quantum jumps among the unperturbed levels (9) [8, 10, 11, 12, 13.

It might be a benefit for the reader to rewrite eq. (30) in terms of $\omega$ and $M$ using $n_{\max }=2 M^{2}$ and $2 M^{2}-m=2\left(M-\omega_{m}\right)^{2}$. One gets:

$$
\Gamma_{m \rightarrow n}=\left\{\frac{1-\exp [-2 \pi]}{1-\exp \left[-4 \pi\left(M-\omega_{m}\right)^{2}+2 \pi\right]}\right\} \exp [-2 \pi(n-m)] .
$$

Eq. (31) can be useful when exploring $n=m+1$ expressions throughout the spectrum as a function of $\omega_{m} / 2 M$.

In any case, we notice from eq. (28) that

$$
\alpha_{m} \simeq 1-\exp [-2 \pi] \simeq 1 \text { for } n_{\max } \gg m
$$

and, for increasing $m$

$$
\alpha_{m} \rightarrow 1^{-} \text {for } m \rightarrow n_{\max }^{-}
$$

with

$$
\alpha_{m}=1 \text { for } m=n_{\max } .
$$

In other words, the dependence of the pre-factor $\alpha$ on the BH quantum level $m$ is in general well approximated by 1 in all the process of $\mathrm{BH}$ evaporation for which our analysis works, included late times in the evaporation process. Clearly, as we are using a semi-classical approximation, deviations could be present at the 
Planck scale. On the other hand, we need a full theory of quantum gravity in order to achieve the Planck scale physics.

The analysis in this work is strictly correct only for $n \gg 1$, i.e. only for excited BHs. This is the reason because we assumed an emission from the ground state to a state with large $n$ in the discussion. On the other hand, a state with large $n$ is always reached at late times, maybe not through a sole emission from the ground state, but, indeed, through various subsequent emissions of Hawking quanta. For the sake of completeness, it is better to explicitly describe in which part of this process needed to reach a large $n$ the description in this paper would start to be valid [42]. Let us consider again an astrophysics BH having an original mass $M$ of the order of 10 solar masses. By inserting $n=10^{6}$ in eq. (9) one gets $E_{n} \simeq 10^{-27} M_{\text {Planck. In other words, the }}$ $\mathrm{BH}$ lost a negligible part of his mass. Thus, we understand that our description is valid for almost all the process of $\mathrm{BH}$ evaporation.

It is also interesting to include a comparison between the importance of the deviation from thermality described in this paper and that due to other phenomena, such as the backscattering of Hawking radiation on the Schwarzschild metric (which is already present at early times in the evaporation) 42. This is a different physical phenomenon due to the existence of a $\mathrm{BH}$ potential barrier 43, 44. Let us consider a scalar field $\Phi$ in the Schwarzschild space-time [43. As this space-time is spherically symmetric, one can separate the Klein-Gordon equation governing the scalar field, i.e. 43

$$
\left(\square+m^{2}\right) \Phi=0,
$$

into spherical harmonics 43

$$
\Phi=\frac{f(r, t)}{r} Y_{l m} \exp [-i \omega t] .
$$

Introducing the Regge-Wheeler tortoise coordinate $x$, defined through the relation 37

$$
\begin{gathered}
x=r+2 M \ln \left(\frac{r}{2 M}-1\right) \\
\frac{\partial}{\partial x}=\left(1-\frac{2 M}{r}\right) \frac{\partial}{\partial r},
\end{gathered}
$$

one gets the resulting radial wave equation as 43 .

$$
\frac{\partial^{2} f}{\partial t^{2}}-\frac{\partial^{2} f}{\partial x^{2}}=V_{l}(r) f
$$

where the potential is 43

$$
V_{l}(r) \equiv\left(1-\frac{2 M}{r}\right)\left[\frac{2 M}{r^{3}}+\frac{l(l+1)}{r^{2}}+m^{2}\right],
$$

and $m$ is the mass of the scalar field [43. Near the BH horizon we get $x \rightarrow-\infty$. As a consequence, the potential falls off exponentially [43]

$$
V \sim \exp \left[\frac{x}{2 M}\right] \text {. }
$$


On the other hand, for $x \rightarrow+\infty$ one gets [43]

$$
\begin{gathered}
V \sim m^{2}\left(1-\frac{2 M}{x}\right) \quad \text { formassive } \Phi \\
V \sim \frac{l(l+1)}{r^{2}} \quad \text { formassless } \Phi .
\end{gathered}
$$

The gravitational field will be partially scattered off on the potential (38) by the incoming waves. Thus, we obtain a superposition of incoming and outgoing waves 43 . As a consequence, the spectrum is not precisely thermal 43.

Although the two phenomena generating deviation from the strict thermal behavior of the Hawking radiation spectrum are different, we recall an interesting recent work where both of them are taken into account [45].

\section{Conclusion remarks}

Assuming the unitarity of BH quantum evaporation [21, 22, 23, 24, 31, 32, 33, 34, 35] and considering the natural correspondence between Hawking radiation and BH QNMs, in a "Bohr-like model" for excited BHs that we recently discussed in a series of papers [10, 11, 12, 13, we have found the intriguing result that the pre-factor of the Parikh and Wilczek probability of emission, although if in general well approximated by 1 , depends on the $\mathrm{BH}$ quantum level $n$. Then, one gets that the emission of Hawking radiation, in the tunneling framework, is completely determined by eqs. (3) and (29).

\section{Acknowledgements}

The author thanks the Referees for useful comments which permitted to improve this paper.

\section{References}

[1] S. W. Hawking, Commun. Math. Phys. 43, 199 (1975).

[2] M. K. Parikh and F. Wilczek, Phys. Rev. Lett. 85, 5042 (2000).

[3] M. K. Parikh, Gen. Rel. Grav. 36, 2419 (2004).

[4] R. Banerjee and B.R. Majhi, JHEP 0806, 095 (2008).

[5] M. Angheben, M. Nadalini, L. Vanzo and S. Zerbini, JHEP 0505, 014 (2005).

[6] M. Arzano, A. J. M. Medved and E. C. Vagenas, JHEP 0509, 037 (2005).

[7] R. Banerjee and B.R. Majhi, Phys. Lett. B 675, 243 (2009).

[8] C. Corda, Ann. Phys. 337, 49 (2013), final version with corrected typos in arXiv:1305.4529v3. 
[9] S. W. Hawking, Phys. Rev. D 14, 2460 (1976).

[10] C. Corda, Int. Journ. Mod. Phys. D 21, 1242023 (2012).

[11] C. Corda, JHEP 1108, 101 (2011).

[12] C. Corda, Eur. Phys. J. C 73, 2665 (2013).

[13] C. Corda, S. H. Hendi, R. Katebi, N. O. Schmidt, 06, 008 (2013).

[14] C. Corda, S. H. Hendi, R. Katebi, N. O. Schmidt, Adv. High En. Phys. 527874 (2014).

[15] M. Maggiore, Phys. Rev. Lett. 100, 141301 (2008).

[16] L. Motl, Adv. Theor. Math. Phys. 6, 1135 (2003).

[17] J. York Jr., Phys. Rev. D28, 2929 (1983).

[18] N. Bohr, Zeits. Phys. 2, 423 (1920).

[19] S. Hod, Gen. Rel. Grav. 31, 1639 (1999).

[20] S. Hod, Phys. Rev. Lett. 814293 (1998).

[21] B. Zhang, Q.-Y. Cai, L. You,, and M. S. Zhan, Phys. Lett. B 675, 98 (2009).

[22] B. Zhang, Q.-Y. Cai, M. S. Zhan, and L. You, Ann. Phys. 326, 350 (2011).

[23] X.-K. Guo, Q.-Y., Cai, Int. Journ. Theor. Phys., published online, DOI 10.1007/s10773-014-2095-8 (2014).

[24] B. Zhang, Q.-Y. Cai, M. S. Zhan, and L. You, Int. Journ. Mod. Phys. D $22,1341014(2013)$.

[25] N. Bohr, Philos. Mag. 26,1 (1913).

[26] N. Bohr, Philos. Mag. 26 , 476 (1913).

[27] R. J. Adler, P. Chen and D. I. Santiago, Gen. Rel. Grav. 3, 2101 (2001).

[28] S. Shankaranarayanan, Mod. Phys. Lett. A 23, 1975-1980 (2008).

[29] J. Zhang, Phys. Lett. B 668, 353-356 (2008).

[30] J. D. Bekenstein, Lett. Nuovo Cim. 11, 467 (1974).

[31] J. M. Maldacena, Adv. Theor. Math. Phys 2, 231 (1998).

[32] L. Susskind, The Black Hole War: My Battle with Stephen Hawking to Make the World Safe for Quantum Mechanics, Little, Brown and Company (2008). 
[33] S. W. Hawking, Phys. Rev. D 72, 084013 (2005).

[34] S. W. Hawking, arXiv:1401.5761 (2014).

[35] Samir D. Mathur, arXiv:1108.0302v2 (hep-th).

[36] B. Datta and A. N. Singh, Ind. Jour. Hist. Sci. 28, 103 (1993).

[37 ] C. Corda, Invited Review for the Advances in High Energy Physics Special Issue "Dark Atoms and Dark Radiation", edited by Maxim Khlopov, Konstantin Belotsky, Jean-René Cudell and Chris Kouvaris (2015), pre-print in arXiv:1503.00565.

[38] S. W. Hawking, "The Path Integral Approach to Quantum Gravity", in General Relativity: An Einstein Centenary Survey, eds. S. W. Hawking and W. Israel, (Cambridge University Press, 1979).

[39] C. Corda, Ann. Phys. 353, 71 (2015).

[40] Q. Q. Jiang and Y. Han, Phys.Lett. B 718584 (2012).

[41] S. Z. Yang, H. L. Li, Q. Q. Jiang and M. Q. Liu, Sci China-Phys. Mech. Astron. 50, 2249 (2007).

[42] Private communication with the referees.

[43] D. Deeg, "Quantum Aspects of Black Holes", edoc.ub.unimuenchen.de/.../1/Deeg_Dorothea.pdf.

[44] V. Mukhanov, A. Wipf, A. Zelnikov, Phys. Lett. B 332283 (1994).

[45] R. Torres, F. Fayos, O. Lorente-Espin, Phys. Lett. B 720, 198 (2013). 